\section{Treatment relapsed subcuta- neous panniculitis-like T-cell lymphoma together HPS by Cyclosporin A}

\author{
Ren'An Chen, Li Liu, Ying Min Liang \\ Department of Haematology, Tang du \\ Hospital, Fourth Military Medical \\ University, Xi'an, China
}

\begin{abstract}
A 25-year-old man was diagnosised subcutaneous panniculitis-like T-cell lymphoma (SPTCL) through biopsy of a nodule from the anterior chest. After the treatment with prednisone $90 \mathrm{mg} 3$ weeks and tapered off in 1 month, the disease released, but relapsed together with symptions of hemophagocytic syndrome eight months after the termination of prednisone. CHOEP recipe was given but with unsatisfactory result until cyclosporine was prescribed. Cyclosporine was removed 6 months later. There is no evidence of clinical relapse 1 year later. This case suggest that cyclosporine could be a selectable treatment even in relapsed SPTCL.
\end{abstract}

\section{Introduction}

According the latest iteration of the WHO classification, subcutaneous panniculitis-like T-cell lymphoma(SPTCL) is refer to $\alpha \beta$ T-cell derivation cytotoxic tumor. ${ }^{1}$ Multiple subcutaneous nodules of varying size or necrotic, mostly located on the trunk, and extremities, may be misdiagnosed as panniculitis because of it's deceptively benign appearance., ${ }^{2,3}$ SPTCL affects both man and women with a broad age range, but rarely in children younger than 2 years. ${ }^{4}$

Some literatures about the treatment of SPTCL have been published, but no scheme was involved in relapsed SPTCL. Herein, we introduce a relapsed SPTCL was successfully cured by cyclosporin A.

\section{Case Report}

A 25-year-old man presented with a 1-month history of recurrent, multiple, reddish skin lesions and nodules on the anterior chest surface together with low-grade fever, general fatigue and anorexia. A deep punch biopsy of a nodule from the anterior chest verified the diagnosis of SPTCL, immunohistochemistry showed the neoplastic cells were positive for: LCA, CD3, CD8, granzyme B, and perforin, and negative for CD56 and CD20. The symptions improved in 4 days and disappeared in 1 week after prednisone $90 \mathrm{mg}$ daily was given intermittently -which maintained 3 weeks and was tapered off in 6 weeks. But the same lesions and nodules was founded in the same location with 4 -kg weight loss eight months after the termination of prednisone. The patient was admitted to our hospital with the examination of pale, and febrile(temperature of $39^{\circ} \mathrm{C}$ ). Local skin examination revealed multiple erythematous, tender, and firm subcutaneous nodules of variable size $(1-1.5 \mathrm{~cm})$ on the anterior chest. Physical examination, chest $\mathrm{X}$ ray, CT scan of abdomen, and bone marrow studies, revealed no other site involvement. There was moderate hepatosplenomegaly. Mucous membranes were free of lesions. Laboratory investigations showed pancytopenia, an elevated erythrocyte sedimentation rate $(66 \mathrm{~mm} / \mathrm{h})$, normal renal function tests, abnormal hepatic function tests (alanine aminotransferase 188 U/L, aspartate aminotransferase $236 \mathrm{U} / \mathrm{L}$, alkaline phosphatase $635 \mathrm{U} / \mathrm{L}$, total bilirubin 98 $\mu \mathrm{mol} / \mathrm{L}$, conjugated bilirubin $22 \mu \mathrm{mol} / \mathrm{L}$, and high triglycerides $465 \mathrm{mg} / \mathrm{dL}$. Prothrombin time and activated partial thromboplastin time is $26 \mathrm{sec}$., $48 \mathrm{sec}$. respectively; fibrinogen is 74 $\mathrm{mg} / \mathrm{dL}$; and positive fibrinogen degradation products were also noted. Throat, midstream urine, and blood culture results were negative. Serologic tests for syphilis, HIV, and hepatitis $\mathrm{B}$ and $\mathrm{C}$ viruses were negative. Tuberculin and Coombs tests were negative. The $\alpha 1$-antitrypsin level was normal. Antinuclear and antismith antibodies, rheumatoid factor, and cryoglobulins were negative. CT showed moderate hepatosplenomegaly. Bone marrow aspirate showed hemophagocytosis of red blood cells and neutrophils by histiocytes.

A diagnosis of SPTCL together HPS was made and a CHOEP recipe -cyclophosphamide 〔750 mg/m², intravenously (i.v.), doxorubicin (50 $\mathrm{mg} / \mathrm{m}^{2}$ i.v.), vincristine (2 $\mathrm{mg}$ i.v.) all given on day 1 and prednisone (90 mg/day per os) given on days $1-5$, etoposide $100 \mathrm{mg} / \mathrm{m}^{2}$ i.v. on days 1-3 were given. Fever and skin lesions resolved within 2 weeks after the treatment but relapsed in 1 week later. A FMD regimen was given but with no effect. Then cyclosporine A $200 \mathrm{mg}$ together with prednisone $90 \mathrm{mg}$ per day were added. The temperature become normal and skin lesions and nodules improved 1 week later. Then prednisone was removed (tapered off in 1 month) and only cyclosporine A $200 \mathrm{mg}$ was taken every day. After 1 month, this patient was discharged with all the systemic symptoms and skin lesions resolved. Maintenance dose of cyclosporine A was continued in 6 months and then removed. The patient's condition remained in remission at 12 -month follow-up
Correspondence: YingMin Liang, Department of Haematology, Tang du Hospital, Fourth Military Medical University, Xi'an 710038, China.

E-mail: liangym@fmmu.edu.cn

Key words: relapsed, subcutaneous panniculitislike T-cell lymphoma, cyclosporin A.

Contributions: Ren'An Chen, Li Liu and Ying Min Liang co-designed the study, enrolled patients, analyzed results and wrote the manuscript.

Conflict of interest: the authors report no conflicts of interest.

Received for publication: 29 September 2010. Accepted for publication: 23 November 2010.

This work is licensed under a Creative Commons Attribution 3.0 License (by-nc 3.0).

CC Copyright R'A. Chen et al., 2010

Licensee PAGEPress, Italy

Hematology Reports 2010; 2:e9

doi:10.4081/hr.2010.eg

with a normal blood count; there was no evidence of clinical relapse.

\section{Discussion}

The distinctive clinicopathological features of a T-cell lymphoma involving the subcutaneous tissue were firstly described by Gonzalez in 1991. ${ }^{2}$ It have been identified that it's drived from cytotoxic $\mathrm{T}$ lymphocytes and named SPTCL in the Revised European American classification of lymphoid neoplasms. ${ }^{5}$ Two distinct subtypes of SPTCL have been classified by the cellular origins: $\alpha \beta$ and $\gamma \delta$ T-cell derivation., ${ }^{4,6}$ Now, SPTCL is specially refer to the cytotoxic tumor of $\alpha \beta$ T-cell derivation., ${ }^{1,7}$

In SPTCL, subcutaneous fat is involved that make panniculitis-like appearance. Morphologically, the lymphoma infiltrate involves the lobules of the subcutaneous tissue, resulting in a typical lobular-panniculitis-like pattern. The lymphoma cells are small to medium in size with moderate pale cytoplasm; the nuclei are round to irregular and often hyperchromatic. The neoplastic cells are generally confined to subcutaneous tissue and frequently infiltrate individual fat cells with a rim-like arrangement at the cell border. Dermal and epidermal involvement are generally absent. ${ }^{6}$ Tumor cells are most often CD8 ${ }^{+}$cytotoxic lymphocytes expressing one or more cytotoxic granule proteins including TIA-1, granzyme B, and perforin and the $\alpha \beta$ TCR. CD56 are are negetive in most cases.

SPTCL typically presents with multiple subcutaneous masseson the extremities and/or 
trunk. Clinical symptoms include malaise, fatigue, myalgia and weight loss. manifestations of systemic involvement are fever, hepatosplenomegaly, mucosal ulcers and sometimes serosal effusions. The clinic course may present in an indolent manner but to be aggressive when the hemophagocytic syndrome(HPS) happen. HPS, a presenting feature in $37 \%$ of patients with SPTCL, ${ }^{7}$ is characterized by fever, cytopenia, splenomegaly, abnormal liver function, and the pathologic finding of hemophagocytosis (phagocytosis of erythrocytes, leukocytes, platelets, and their precursors bymacrophages) in bone marrow. ${ }^{8}$ The appearance of HPS was thought to be related to cytokine and chemokine production by the malignant cells, perhaps in a setting of comprised cytolytic function. This patient have pancytopenia, liver function abnormalities, hepatosplenomegaly, pleural effusion, hypertriglyceridemia, and coagulopathy, in keeping with the diagnosis of relapsed SPTCL complicated by HPS.

Treatments such as systemic chemotherapy, radiotherapy, ${ }^{9}$ stem cell transplantation ${ }^{10,11}$ and limb amputation ${ }^{12}$ have been used since this disease described. NO standard treatment strategy have been made because of the rarity of the disease. First of all, systemic steroids or other immunosuppressive agents should be applied in SPTCL without associated HPS, whereas radiotherapy should be considered in cases of solitary skin lesions. Multi-agent chemotherapy may be required in cases with progressive disease not responding to immunosuppressive therapy or in cases with HPS, ${ }^{7}$ but anthracycline-based combination chemotherapy (CHOP or CHOP-like) were reported to be with unsatisfactory result. ${ }^{13}$ Other materials, such as Denileukin diftitox, a recombinant fusion protein that combines human interleukin 2 and diphtheria toxin, have been applied on SPTCL with challenging outcome. ${ }^{14}$ Prednisone have been applied on SPTCL with optimistical result although some still be invalid. In this patient, prednisone made a period of 8 months tumor release but be ineffective ultimately. Even HPS, a syndrome was reported can be cureed by prednisone, ${ }^{15}$ emerging at the eighth month of the application of prednisone, proved the treatment was aborted. CHOP together with etoposide, another cytostatic drug which was verified to be effective to HPS,$^{16}$ so called CHOEP, made no significant effect. SPTCL persisted until cyclosporine was prescribed.

Cyclosporine, a calcineurin inhibitor, is a potent immunosuppressant that reduces the production of several growth factors. In spite of the significantly worse prognosis of SPTCL complicated by HPS, ${ }^{17}$ cyclosporine have been verified to be with magical effect in a few reports. $^{18,19}$ The mechanism of action of cyclosporin in SPTCL is also down-regulation of cytokines. In this patient, cyclosporine (200 $\mathrm{mg} / \mathrm{d}$ ) made the symptoms improved within 1 week and resolved in 1 month. After 6 months, cyclosporine was removed. There is no evidence of clinical relapse 1 year later.

\section{Conclusions}

There is not standard treatment to SPTCL because of the rarity of the disease, especially to relapsed phase. We successfully curred a patien with relapsed SPTCL complicated by HPS. This report suggest that cyclosporine A may be still an effictive therapy when this disease relapsed.

\section{References}

1. Willemze R, Jaffe ES, Burg G, et al. WHOEORTC classification for cutaneous lymphomas. Blood 2005;105:3768-85.

2. Gonzalez CL, Medeiros LJ, Braziel RM, Jaffe ES. T-cell lymphoma involving subcutaneous tissue: a clinicopathologic entity commonly associated with hemophagocytic syndrome. Am J Surg Pathol 1991;15:1727.

3. Burg G, Dummer R, Wilhelm M, et al. A subcutaneous delta-positive T-cell lymphoma that produces interferon gamma. $\mathrm{N}$ Engl J Med 1991;325:1078-81.

4. Salhany KE, Macon WR, Choi JK, et al. Subcutaneous panniculitis-like T-cell lymphoma: clinicopathologic, immunophenotypic, and genotypic analysis of alpha/beta and gamma/delta subtypes. Am J Surg Pathol 1998;22:881-93.

5. Harris NL, Jaffe ES, Stein H, et al. A revised European-American classification of lymphoid neoplasms: a proposal from the International Lymphoma Study Group. Blood 1994;84:1361-92.

6. Toro JR, Liewehr DJ, Pabby N, et al. Gamma-delta T-cell phenotype is associated with significantly decreased survival in cutaneous T-cell lymphoma. Blood 2003; 101:3407-12.

7. Willemze R, Dreyling M. ESM0 Guidelines Working Group.Primary cutaneous lymphomas: ESMO Clinical Practice Guidelines for diagnosis, treatment and follow-up. Ann Oncol 2010;21:v177-80.

8. Fisman DN. Hemophagocytic syndromes and infection. Emerg Infect Dis 2000; p6:601-8.

9. Perniciaro C, Zalla MJ, White JW, Menke DM. Subcutaneous T-cell lymphoma. Arch Dermatol 1993;129:1171-6.

10. Hashimoto H, Sawada K, Koizumi K, et al. Effective CD34+selected autologous peripheral blood stem cell transplantation in a patient with subcutaneous panniculitic T-cell lymphoma (SPTCL) transformed into leukemia. Bone Marrow Transplant 1999;24:1369-71.

11. Leonard GD, Hegde U, Butman J, et al. Extraoular muscle palsies in subcutaneous panniculitis-like T-cell lymphoma. J Clin Oncol 2003;21:2993-5.

12. Haycox CL, Back AL, Raugi GJ, PiepkornM. Subcutaneous T-cell lymphoma treated with systemic chemotherapy, autologous stem cell support, and limb amputation. J Am Acad Dermatol 1997;37:832-5.

13. Go RS, Wester SM. Immunophenotypic and molecular features, clinical outcomes, treatments, and prognostic factors associated with subcutaneous panniculitis-like T-cell lymphoma: a systematic analysis of 156 patients reported in the literature. Cancer 2004;101:1404-13.

14. McGinnis KS, Shapiro M, Junkins-Hopkins JM, et al. Denileukin diftitox for the treatment of panniculitic lymphoma. Arch Dermatol 2002;138:740-2.

15. Janka G, zur Stadt U. Familial and acquired hemophagocytic lymphohistiocytosis.Hematology Am Soc Hematol Educ Program 2005:82-8.

16. Ogasawara T, Kawauchi K, Yasuyama M, Ohkawa S. Successful use of etoposide in an elderly patient with chronic recurrent hemophagocytic syndrome. Nippon Ronen Igakkai Zasshi 2003;40:160-6.

17. Willemze R, Jansen PM, Cerroni L, et al. Subcutaneous panniculitis-like T-cell lymphoma: definition, classification and prognostic factors: an EORTC Cutaneous Lymphoma Group study of 83 cases. Blood 2008;111:838-45

18. Iwasaki T, Hamano T, Ogata A, et al. Successful treatment of a patient with febrile, lobular panniculitis (WeberChristian disease) with oral cyclosporin A: implications for pathogenesis and therapy. Intern Med 1999;38:612-4.

19. Rojnuckarin P, Nakorn TN, Assanasen T, et al. Cyclosporin in subcutaneous panniculitis-like T-cell lymphoma. Leuk Lymphoma 2007;48:560-3. 